Hacia una nueva epistemología de la psiquiatría.

German E. Berrios

Buenos Aires: Editorial Polemos, 2011, 312 págs.

\title{
Hacia una nueva epistemología de la psiquiatría
}

Rogelio Luque*

Histórica y epistemológicamente, la psiquiatría es una disciplina relativamente nueva. Nacida durante el siglo XIX bajo la tutela de la medicina, se edificó sobre las ciencias sociales y las ciencias naturales. Mientras las primeras proporcionan los criterios sociales, morales y estéticos para la categorización y la clasificación de las conductas anómalas, las segundas intentan inscribir dichas conductas en el cerebro. Inicialmente, la investigación pretende comprender por qué la sociedad considera "desviadas" determinadas conductas en cada periodo histórico $\mathrm{y}$, posteriormente, conocer cuáles son las razones que llevan a algunas personas a desarrollar perturbaciones mentales. Una vez establecidas las conductas anómalas por los agentes sociales, entran en escena las ciencias naturales tratando de vincular las configuraciones (conductas) al cuerpo (cerebro). Obviamente, las

* Universidad de Córdoba. (Córdoba, España) 


\section{RESENHAS BIBLIOGRÁFICAS}

ciencias naturales por sí solas no pueden crear nuevas categorías/definiciones de "trastorno mental" y, en consecuencia, las ciencias humanas tienen primacía epistemológica. Por su naturaleza "híbrida", la psiquiatría se diferencia de otras especialidades médicas y esta singularidad debería regir su enseñanza, su investigación y su práctica.

Para tratar de responder a los problemas y desafíos conceptuales planteados por esta peculiaridad de la psiquiatría, el profesor Germán E. Berrios, catedrático de Epistemología de la Psiquiatría de la Universidad de Cambridge (Reino Unido) y el grupo de psiquiatras, historiadores y filósofos aglutinado a su alrededor y conocido como la Escuela Psicopatológica de Cambridge, han ido desarrollando y perfeccionando durante los últimos 30 años una "nueva epistemología de la psiquiatría". La producción científica del profesor Berrios es extraordinaria e incluye 15 libros sobre neuropsiquiatría, psicopatología descriptiva e historia conceptual de la psiquiatría y más de 400 artículos. Entre los primeros destacan The History of Mental Symptoms (edición en español: Historia de los Sintomas de los Trastornos Mentales, Fondo de Cultura Económica, 2008) y que en 1997 ganó el British Medical Journal Prize y A History of Clinical Psychiatry, editado junto a Roy Porter y recientemente traducido al español y al portugués. Además, es editor de la prestigiosa revista History of Psychiatry (que fundó en 1989 también junto a Roy Porter).

Buen ejemplo del nivel de excelencia alcanzado en la superación de los escollos conceptuales de la psiquiatría lo constituye el último libro del profesor Berrios, Hacia una nueva epistemología de la psiquiatría, publicado en español por la Editorial Polemos antes de su edición en inglés. La obra consta de cuatro partes: 1) filosofía y epistemología de la psiquiatría; 2) el lenguaje de la psiquiatría; 3) objetos de la psiquiatría; y 4) datos de la psiquiatría. La primera parte afronta temas como la historia de las filosofías de la psiquiatría, la epistemología de la psiquiatría, los conceptos de etiología, riesgo, comorbilidad y clasificación en psiquiatría, y los modelos y las analogías. La segunda se dedica a la epistemología de la psicopatología como lenguaje de la psiquiatría, mientras que la sección los objetos de la psiquiatría aborda el concepto y la epistemología de los síntomas mentales, la forma, el contenido y la formación de los mismos y su consideración como "síntomas híbridos". Además, trata sobre las fenocopias conductuales, la localización cerebral de los síntomas mentales y la epistemología de las emociones. La última sección se reserva a la epistemología de los datos psiquiátricos y las escalas de evaluación, concluyendo con un capítulo dedicado al concepto de evidencia y su aplicación a la psiquiatría (basada en la evidencia), que resulta un demoledor desmontaje de lo que para el profesor Berrios supone un "truco destructivo" y cuya aplicación a la práctica de la psiquiatría puede causar un daño irreparable si no se remedia pronto. 


\section{La psiquiatría como disciplina híbrida}

Todas las ciencias conciernen a un objeto que se supone específico. En las ciencias naturales (botánica, zoología) el objeto se identifica mejor que en las humanidades (historia del arte, antropología social). Esta facilidad en la identificación de los objetos de las ciencias naturales generalmente se explica alegando que estos son ontológicamente independientes. Actualmente, la psiquiatría biológica defiende que los objetos de la psiquiatría incumben a las ciencias naturales. Desde esta perspectiva, los síntomas y las enfermedades mentales serían clases naturales y podrían reducirse a modificaciones cerebrales patológicas. Este enfoque ha perjudicado a la psiquiatría clínica y ha descuidado el pensamiento psicopatológico y la investigación conceptual. Para el profesor Berrios es imprescindible (y urgente) desvelar los motivos por los que se ha llegado a esta situación, y exige realizar una revisión conceptual de la psiquiatría mediante el análisis histórico y epistemológico conjunto, abordando temas fundamentales como la naturaleza del conocimiento psiquiátrico, sus fuentes y origen; su estabilidad, legitimidad, y persistencia; la formación de los objetos psiquiátricos y su relación con el cerebro.

Tradicionalmente, para el discernimiento del significado de la locura en sus distintos contextos (histórico, social, biológico y psicológico) se han construido narrativas específicas e independientes. Esto ha provocado la división de la psiquiatría en patrones concretos y paralelos (psiquiatría biológica, psicodinámica, social) sin que exista una conexión entre ellos. En consecuencia, se ha escatimado la investigación sobre cómo otros contextos podrían ajustarse al marco conceptual dominante. Por ejemplo, las narrativas biológicas raramente exploran la contribución de los factores psicosociales en las manifestaciones de la enfermedad mental. Una de las tareas centrales de la psiquiatría consiste en comprender cómo interactúan los diversos patrones de estas narrativas. El objetivo fundamental sería clasificar de qué forma se entrelazan los distintos aspectos de las ciencias humanas y las naturales en los diversos objetos de estudio. Las cuestiones derivadas de esta profunda amalgama entre ambas ciencias son complejas, y no pueden despacharse superficialmente afirmando que la psiquiatría necesita "diferentes modelos" o recurriendo a un "pluralismo convencional" como el modelo biopsicosocial.

La original propuesta del profesor Berrios consiste en considerar los síntomas mentales como objetos híbridos, constituidos por una amalgama especial de señales biológicas y configuradores personales, familiares, sociales y culturales. Esto no quiere decir que los objetos híbridos deban considerarse como una simple combinación de objetos físicos y abstractos. Su naturaleza está 


\section{RESENHAS BIBLIOGRÁFICAS}

marcada por la configuración que realizan las personas que afrontan una experiencia compleja y desconcertante y, por tanto, están inculcados de poderosas fuerzas cognitivas, emocionales y volitivas. La mayoría de los síntomas mentales comienza como señales cerebrales que al penetrar en la conciencia se experimentan como proto-experiencias. Para que esa experiencia pueda comunicarse previamente debe configurarse. Pero el sujeto carece de patrones emocionales y cognitivos previos que permitan configurar estas experiencias extrañas. Por eso provocan perplejidad y temor. En ocasiones, mediante un complejo proceso de negociación con el médico, el paciente consigue transmitirlas verbalmente. Esto constituye el síntoma mental. En este sentido, el síntoma mental no puede explicarse por ninguna estructura neurobiológica sino por la envoltura semántica y simbólica que les confiere un valor comunicativo específico.

\section{¿Por qué una "nueva epistemología” de la psiquiatría?}

La epistemología estudia el origen y la revisión de los conceptos y la legitimación del conocimiento. Para realizar adecuadamente su cometido debe mantenerse independiente de los objetos a estudiar, sin adjudicar a ninguna ciencia la primacía en la búsqueda de la autentificación, intentando por el contrario desvelar el papel que desempeñan los conceptos en la formación del conocimiento.

Si la psiquiatría se define como una disciplina teórica y práctica cuya estructura epistemológica está a caballo entre las ciencias naturales y las humanidades, la epistemología de la ciencia y la epistemología de la medicina carecen de los instrumentos específicos para desentrañar los "objetos" exclusivos a su naturaleza. Por tanto, la psiquiatría está obligada a construir su propia epistemología. Esta consistirá en una "epistemología regional", que explore específicamente la estructura y la legitimidad del conocimiento psiquiátrico y de los objetos de la psiquiatría. Deberá, asimismo, desentrañar sus diversos elementos (valores, componentes orgánicos y símbolos) enraizados en diferentes fuentes de conocimiento y que están en constante interacción. En definitiva, la epistemología regional y específica de la psiquiatría tiene que dilucidar la naturaleza de los objetos psiquiátricos, determinando sus fuentes constitutivas y las magnitudes de cada una de ellas para elegir la forma más válida de gestionar dichos objetos.

La nueva epistemología de la psiquiatría debe recurrir a métodos adicionales como la historia, la filosofía, la psicología social, la antropología, la lingüística y la hermenéutica. Concretamente, la filosofía de la psiquiatría juega un papel esencial en el análisis de la naturaleza de los síntomas mentales ("No se puede concebir una psiquiatría sin filosofía", afirma el profesor Berrios). Pero 
esta no debe realizarse con un estilo centrípeto, que utiliza sistemas filosóficos establecidos (la fenomenología, el empirismo lógico, la filosofía analítica) para identificar, configurar, clasificar y resolver las distintas cuestiones de la psiquiatría. Esta aproximación centrípeta ha sido utilizada tanto por filósofos como por psiquiatras. En el primer caso, corren el riesgo de convertirse en escuderos de la psiquiatría biológica con la misión de proporcionar apoyo filosófico a sus fundamentos teóricos y dictados ideológicos. En el segundo caso, la mayoría de las propuestas se limita a combinar fragmentos de diferentes sistemas filosóficos en una suerte de sincretismo o de pluralismo. Por el contrario, Berrios defiende una aproximación centrífuga, surgida del mismo corazón de la psiquiatría, que conceptúe los problemas desde dentro y elabore un metalenguaje específico para analizarlos. No todos los problemas que la psiquiatría debe analizar son del mismo tipo. Para aquellos extrínsecos a la psiquiatría (como la definición de lo mental, la relación mente-cuerpo, la distinción entre causas y razones o la reducción interdisciplinar), pueden compartirse los métodos comunes a la filosofía de la ciencia y de la mente. Sin embargo, otras cuestiones intrínsecas a la propia disciplina (qué son los síntomas mentales y cómo se generan, cómo explicar la heterogeneidad de éstos, qué es un trastorno mental, cómo diagnosticarlo y clasificarlo) no pueden ser solventadas con soluciones preestablecidas y precisan de nuevas y originales propuestas que tengan en cuenta la naturaleza de la psiquiatría y de su objeto de estudio.

La psiquiatría sigue siendo una disciplina descriptiva. Su especial naturaleza y su amplio alcance conceptual requieren nuevas elaboraciones teóricas y distintos planes de investigación. En este sentido, Hacia una nueva epistemología de la psiquiatría constituye una lectura imprescindible para psiquiatras, filósofos e historiadores. Algunos de los capítulos del libro han aparecido previamente en diversas publicaciones. Sin embargo, reunidos en un solo volumen permite una lectura encadenada de los diversos temas que adquieren así una dimensión y un significado especiales. Aquellos que estén familiarizados con el trabajo del profesor Berrios no se sorprenderán de su erudición y de su brillantez intelectual. Se trata de una obra original y enriquecedora, que incita a seguir profundizando en los diversos filones epistemológicos de los síntomas mentales, como pueden ser la comprensión hermenéutica, la clasificación de los síntomas o su inscripción cerebral primaria y secundaria. Para el profesor Berrios y la Escuela de Cambridge la elaboración de una nueva Epistemología de la psiquiatría constituye una necesidad teórica, pero sobre todo resulta un compromiso moral con nuestros pacientes. Como tantas veces ha repetido Germán Berrios, los enfermos mentales son la única razón de nuestra existencia como psiquiatras clínicos. Ellos merecen que les proporcionemos nuevas respuestas terapéuticas a sus padecimientos. 


\section{Referencias}

Berrios, G.E. (2011). Psiquiatría y sus objetos. Revista de Psiquiatría y Salud Mental (Barc.), 4, 179-182.

Marková, I.S., Berrios, G.E. (2012). Epistemology of Psychiatry. Psychopatho$\log y, 45,220-227$.

Citação/Citation: Luque, R. (2015, setembro). Resenha do livro Hacia una nueva espistemología de la psiquiatría. Revista Latinoamericana de Psicopatologia Fundamental, 18(3), 566-571.

Editor do artigo/Editor: Profa. Dra. Sonia Leite

Recebido/Received: 15.3.2015/ 3.15.2015 Aceito/Accepted: 29.4.2015 / 4.29.2015

Copyright: (C) 2009 Associação Universitária de Pesquisa em Psicopatologia Fundamental/ University Association for Research in Fundamental Psychopathology. Este é um artigo de livre acesso, que permite uso irrestrito, distribuição e reprodução em qualquer meio, desde que o autor e a fonte sejam citados / This is an open-access article, which permits unrestricted use, distribution, and reproduction in any medium, provided the original authors and sources are credited.

\section{Rogelio LuQue}

Professor titular de Psicologia Médica e Psiquiatria na Universidad de Cordoba, Espanha. e-mail: rogelioluque@uco.es 